\title{
Case Report of a Successful Hair Transplantation in a Patient with Lichen Planopilaris/Frontal Fibrosing Alopecia Due to Incidental Chemotherapy for Breast Carcinoma
}

\author{
Allison L. Limmer BS BA ${ }^{a}$, Jennifer Krejci-Manwaring $M^{b}$, Bobby L. Limmer MD $^{b}$ \\ ${ }^{a}$ McGovern Medical School at UTHealth, Houston, TX \\ ${ }^{\mathrm{b}}$ The University of Texas Health Science Center at San Antonio, San Antonio, TX
}

\section{ABSTRACT}

Lichen planopilaris (LPP) and its variant frontal fibrosing alopecia (FFA) are disfiguring alopecias that rarely yield satisfactory hair transplantation results. Grafts may grow initially only to be obliterated by re-activation of the disease within the first few months to years post-transplant. Here, we detail the management of a patient who was diagnosed and treated for breast carcinoma after her diagnosis of LPP/FFA. Two years after completion of chemotherapy, the patient presented with quiescence of her LPP and successfully underwent hair transplantation by follicular unit transplantation method. She has maintained the grafts without re-activation of her scarring alopecia for 7 years - a success we attribute to chemotherapy.

\section{CASE REPORT}

A 67-year-old female presented to our office for hair loss evaluation in 2008. She noted progressive hair loss throughout her scalp but most pronounced at her hairline and crown. On exam, there were patches of scant hair growth with background scarring of the crown and mid-scalp with regression of the frontal hairline of approximately $3 \mathrm{~cm}$. There was perifollicular scale and erythema at the crown and mid-scalp but most prominent along the frontal hairline. There were decreased vellus hairs in the crown and few at the hairline, but miniaturization was present in the mid-scalp. No photos were taken, but a biopsy was performed and consistent with late-stage LPP/FFA with a background of androgenetic alopecia. She underwent one treatment with intralesional triamcinolone $2.4 \mathrm{mg} / \mathrm{ml} \times 20 \mathrm{ml}$ to the frontal hairline and vertex scalp and was lost to follow-up.

In 2009 the patient was diagnosed with breast carcinoma and underwent mastectomy and chemotherapy. She was treated with 9 cycles of doxorubicin, cyclophosphamide, and dexamethasone; some cycles included docetaxel. She returned for re-evaluation of her alopecia in 2010, 7 months after completion of chemotherapy. On examination, there was early regrowth of hair from chemotherapy- 
induced anagen effluvium but no erythema or perifollicular scale. Conservative medical management with Rogaine was recommended. Repeat biopsy was not performed. At 1-year follow-up, her hair had recovered back to baseline, as her exam remained virtually unchanged from 2009 : scarring alopecia measuring approximately $5 \times 8 \mathrm{~cm}$ of the crown and $3 \mathrm{~cm}$ regression of the frontal hairline with no scale or erythema - no evidence of active LPP/FFA (Figure 1A, B). The mid-scalp still demonstrated sparse coverage with features of androgenetic alopecia - diffuse thinning, variation in hair shaft caliber, and miniaturization. The rest of the patient's hair shafts were noted to be "fine" in texture. Notably, the patient continued to wear a wig daily since her chemotherapy-induced anagen effluvium.

The patient elected to proceed to transplantation by follicular unit transplantation method (FUT/strip), recognizing the potential for re-activation of her LPP and the limitations relative to fine texture of her donor hair. Her primary goal was to be able to abandon her wig and to reestablish a frontal hairline in a more natural position.

Transplantation was done in April 2011, utilizing approximately 1200 grafts to the frontal hairline concentrated at the frontal tuft and mid-scalp. At 1-year post-transplant, with no medical intervention, the grafts demonstrated stable growth (Figure 2A, B). The patient was re-evaluated yearly until 2014. At that time, she was satisfied with her hair restoration and had abandoned her wig (Figure 3A, B). She did have a mild telogen effluvium in 2014 due to new-onset thyroid disease, but to date there has been no evidence of re-activation of her scarring alopecia.

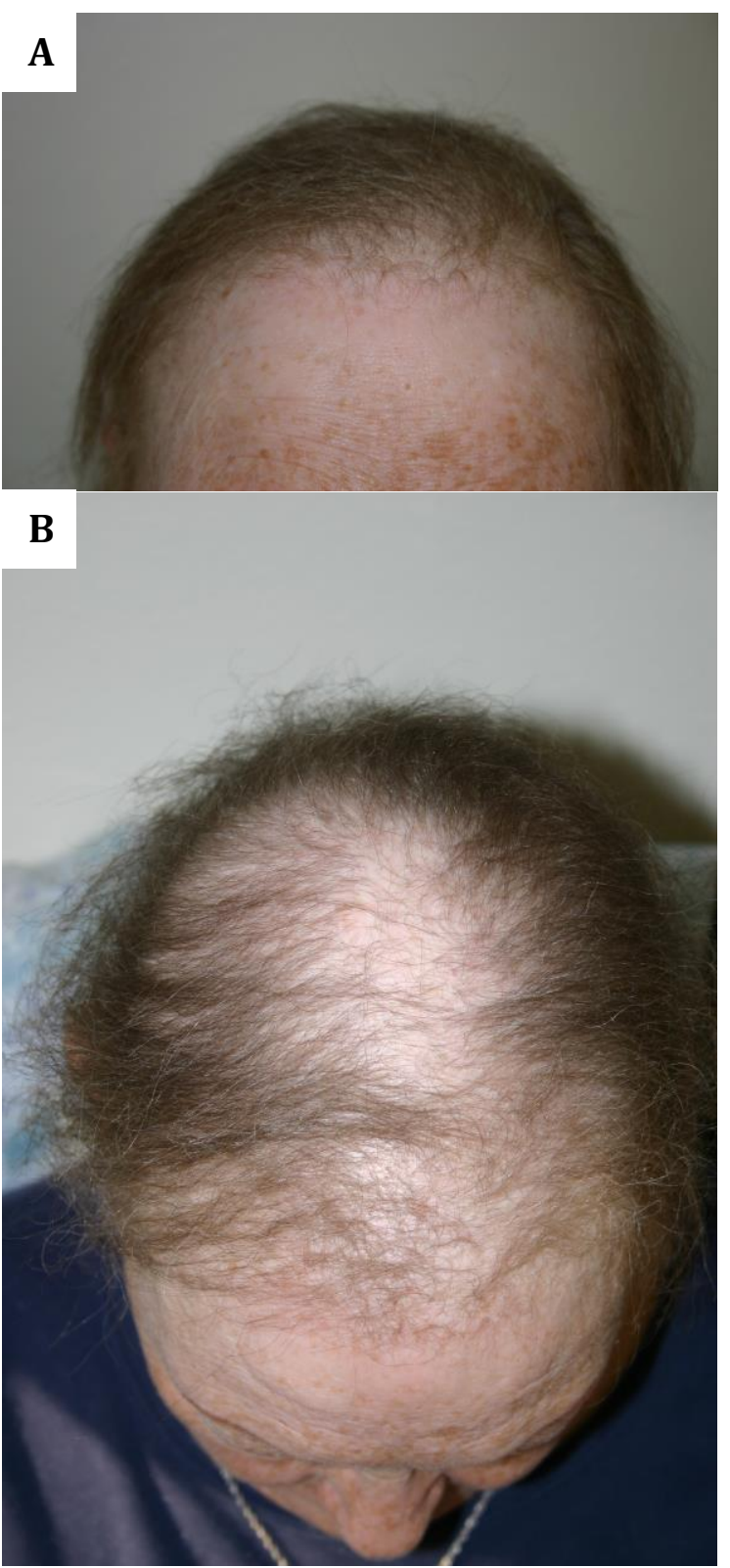

Figure 1. Pre-transplantation: regression of frontal hairline, atrophic porcelain quality of upper forehead skin (A), scant hair growth at crown and mid-scalp but no active disease (B). General "fine" hair texture and diffuse loss in uninvolved scalp. 


\section{SKIN}

\section{A}

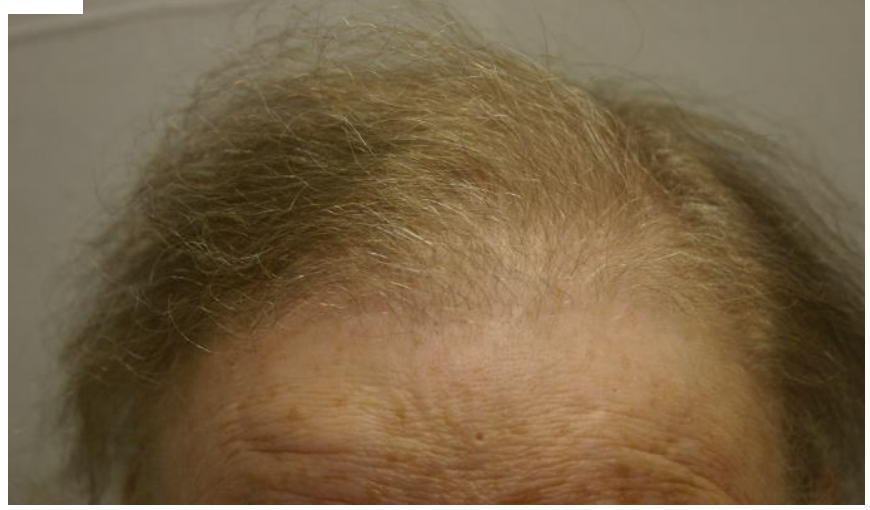

Figure 2. 1-year post-transplant. Lowered, fuller frontal hairline $(A)$ and improvement of hair growth at the crown and mid-scalp (B).

\section{A}

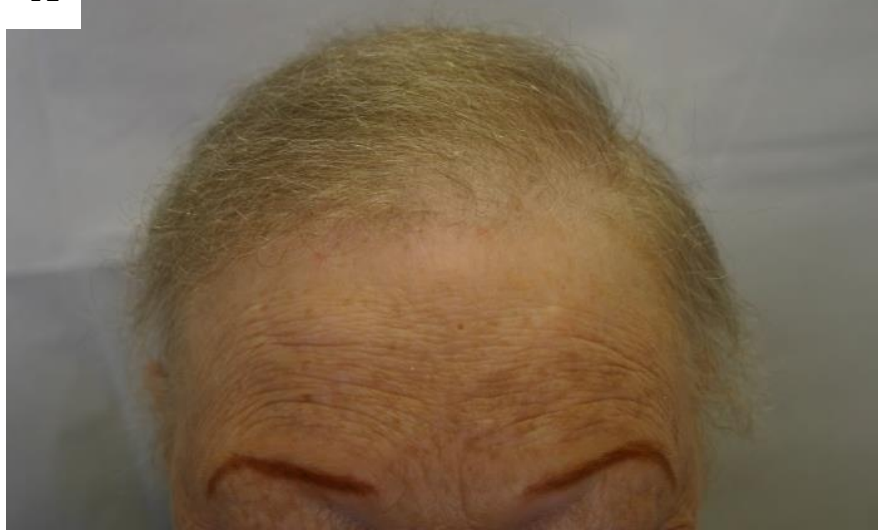

Figure 3. 3-year post-transplant. Persistent improvement in frontal hairline $(A)$ and at crown and mid-scalp (B)
B
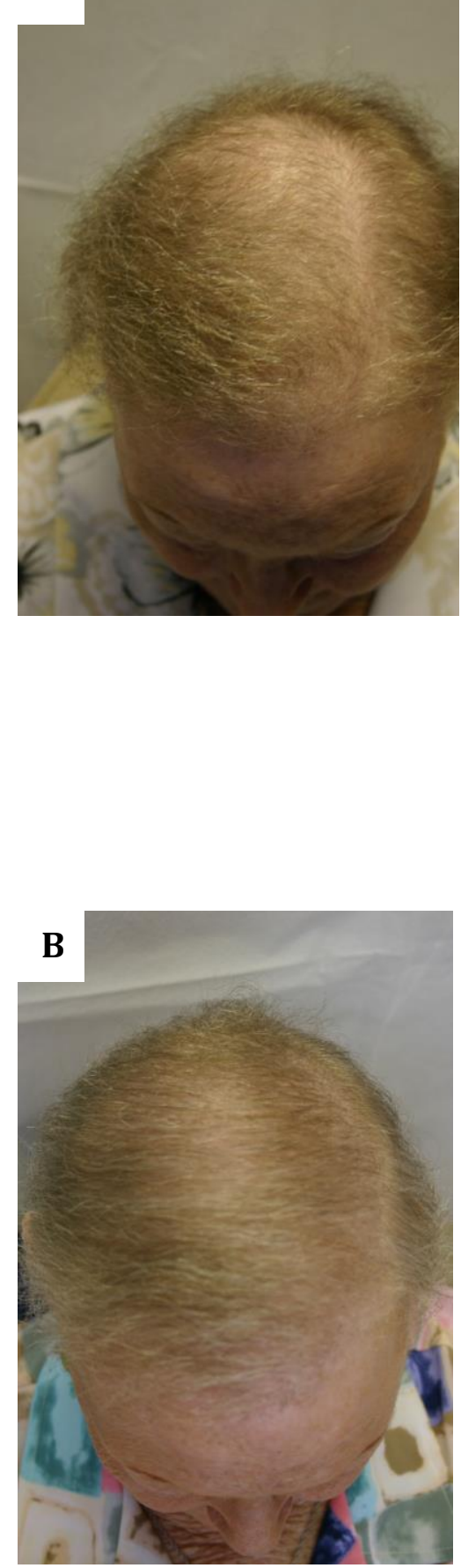


\section{DISCUSSION}

LPP/FFA is a cicatricial alopecia and is especially problematic from the aspects of medical therapy and surgical restoration. Most medical therapy is of limited success, with the main goal being to halt progression of hair loss. Treatments include topical, intralesional, and oral corticosteroids; hydroxychloroquine; tetracyclines; $5 \alpha$ reductase inhibitors; pioglitazone; and immunosuppressants. ${ }^{1}$ Surgical restoration is known to cause re-activation of disease to the implanted follicles and their subsequent obliteration, but reports also suggest hair transplantation can cause primary activation of LPP in patients not previously known to have the disease. ${ }^{2,3}$ It is the authors' opinion that activation of LPP after hair transplantation is more likely undiagnosed LPP that pre-dated the hair transplant. One case report indicates hair transplantation in LPP patients can be successful if scalp and beard hair are combined and platelet rich plasma (PRP) is injected intradermally prior to graft placement. ${ }^{4}$ It is important to note that the follow-up period was only 10 months whereas other reports suggest graft rejection may not start until well over a year after transplantation. In addition, there was more than one variable in the procedure (scalp hair / beard hair / PRP), so it is impossible to know if there were a true causative effect.

Here we present a case from a large hair restoration practice who has had durable remission of LPP/FFA after FUT transplantation. We attribute the remission of the disease process to her incidental chemotherapy for breast cancer. We also raise the question to the medical community, especially our oncology colleagues: is there any potentially safe method by which chemotherapy drugs could be administered topically for this disfiguring cicatricial alopecia?

Conflict of Interest Disclosures: None.

Funding: None.

Corresponding Author:

Allison L. Limmer, BS, BA

6431 Fannin St.

Houston, TX 77030

allison.I.limmer@uth.tmc.edu

References:

1. Whiting DA. Cicatricial alopecia: clinicpathological findings and treatment. Clin Dermatol. 2001;19:211-25.

2. Jiménez F, Poblet E. Is hair transplantation indicated in frontal fibrosing alopecia? The results of test grafting in three patients. Dermatol Surg. 2013;39:1115-8.

3. Donovan J. Lichen planopilaris after hair transplantation: report of 17 cases. Dermatol Surg. 2012;38:1998-2004.

4. Saxena K, Saxena DK, Savant SS. Successful hair transplant outcome in cicatricial lichen planus of the scalp by combining scalp and beard hair along with platelet rich plasma. J Cutan Aesthet Surg. 2016;9:51-5. 